\title{
El estudiante tiene que aprender a evaluar y valorar adecuadamente su propia prestación, al margen ya de las calificaciones
}

\section{Students need to learn to evaluate and assess their own performance, regardless of grades}

\section{Ángela Collados Aís}

Departamento de Traducción e Interpretación, Universidad de Granada angela@ugr.es

\section{INTRODUCCIÓN}

En esta entrevista Ángela Collados Aís nos habla sobre su trabajo como investigadora en el Grupo de investigación ECIS para la evaluación de la calidad en interpretación simultánea. En torno a este tema se plantean algunas cuestiones directamente relacionadas con la docencia, las modalidades de interpretación y la situación laboral de las personas que se dedican a la interpretación en ámbitos tan distintos, desde el punto de vista de la evaluación de la calidad, como la Interpretación en los servicios públicos o la Interpretación de conferencias.

Palabras clave: evaluación de la calidad en interpretación; modalidades de interpretación. y valorar adecuadamente su propia prestación... 


\section{ENTREVISTA}

Fecha: 5/1/2018

Don de Lenguas: Para empezar y para los radioyentes que no lo sepan, queremos hacer una breve introducción sobre la biografía de Ángela Collados Aís. Actualmente, es docente de interpretación en la Facultad de Traducción e Interpretación de la Universidad de Granada desde el año 1987. Ahí imparte interpretación de enlace y de conferencias en la combinación de idiomas alemán-español. Además, tiene una rama investigadora muy amplia; ha dirigido numerosos proyectos de investigación en interpretación y actualmente es la investigadora principal del grupo ECIS de evaluación de la calidad en interpretación simultánea. Además, sus publicaciones, que no son pocas y que recomiendo a los radioyentes que lean, se centran fundamentalmente en el ámbito de la calidad de interpretación y de la docencia. Buenas tardes, Ángela.

Ángela Collados Aís: Muy buenas tardes y muchas gracias por la entrevista.

DDL: Bueno, en primer lugar, queríamos preguntarte, ¿qué es exactamente el ECIS y cuál es vuestra labor?

ACA: Perdona, creo que has dicho ECIS, ¿no?

DDL: Sí.

ACA: Bueno, el grupo de investigación, como bien has dicho, se centra sobre todo en investigar sobre evaluación de la calidad de interpretación simultánea. Es decir, nos focalizamos más, en un principio, en los aspectos no verbales de la evaluación de la calidad. Esos aspectos no verbales que han sido los grandes descuidados, no solamente en interpretación, sino también en general en la comunicación. Porque precisamente creíamos que tenían una gran importancia y se descuidaban, pues se ha centrado más en ellos toda la investigación. En realidad, en el grupo estamos bastantes investigadoras, digo investigadoras porque somos más investigadoras, y tenemos, por así decirlo, distribuidos los distintos factores no verbales que pueden influir en la evaluación de una calidad. Pues, por ponerte un ejemplo, la entonación, pero luego hemos seguido por la fluidez, con la agradabilidad de la voz, con el acento, etc.

DDL: En relación con el lenguaje no verbal y con la entonación, que nos parecen realmente dos puntos muy interesantes a tratar, ¿cómo crees que influyen en los participantes en una situación en la que se requiere interpretación?

ACA: Pues yo creo que afecta muchísimo. Lo primero que hay que tener en cuenta es que la entonación forma parte de lo que se está diciendo, es decir, no es algo aparte. Puede influir de diferentes maneras. En primer lugar, puede producir simplemente

Ángela Collados Ais

El estudiante tiene que aprender a evaluar y valorar adecuadamente su propia prestación...
CLINA

vol. 7-1, June 2021, 57-65

elSSN: 2444-1961

Ediciones Universidad de Salamanca - CC BY-NC-ND 
aburrimiento o que nos formemos una imagen de la persona que está transmitiendo la información que sea como una imagen poco competente en lo comunicativo y de ahí se va extrapolando hacia otros aspectos. Pero incluso creo que las últimas investigaciones han puesto de manifiesto cómo intercepta la entonación en la propia recepción del mensaje. Es decir, hasta ahora se creía que era más bien, por la influencia de esos aspectos no verbales como la entonación, una influencia limitada en el sentido de que puede producir aburrimiento, puede hacer juzgar como poco competente al orador, etc. Pero ahora mismo creo que cada vez está más claro que realmente influye en que se reciba o no se reciba el propio mensaje. Eso lo sabemos, porque realmente la entonación también en la comunicación monolingüe se puede producir así. Es decir, el mensaje llega mejor, o llega, simplemente, cuando se utiliza la entonación adecuada. Cuando no se utiliza la entonación adecuada, se producen problemas en la comunicación. Incluso cuando el orador original es monótono, el intérprete debe evitar caer en esa monotonía. Pero claro, lo mismo que estoy diciendo esto también existe luego el efecto que se llama de «U invertida». Si la entonación es demasiado melodiosa en el intérprete, eso tampoco produce una buena recepción del mensaje, ni siquiera un buen juzgar de la competencia del intérprete, porque el mensaje siempre tiene que estar acorde con esa entonación. O, mejor dicho, la entonación tiene que estar acorde con el mensaje que se transmita. No es lógico que un mensaje que trata sobre temas muy áridos y realmente muy serios se transmita con una voz cantarina. En ese momento tampoco el intérprete está utilizando la entonación adecuada. Por tanto, cuando hablamos de entonaciones, siempre nos referimos a la entonación adecuada al mensaje y a la situación que se trate, lógicamente.

DDL: Claro, y todo en su punto medio, supongo. Porque como tú bien has dicho, si hay una entonación muy melodiosa en un mensaje con un contenido especialmente árido, como bien decías, no encaja. Bueno, aparte de la entonación y del lenguaje no verbal, ¿hay otros parámetros con los que medís la calidad de la interpretación en el ECIS?

ACA: Pues sí. Los parámetros de calidad que nosotros analizamos los basamos en los primeros estudios empíricos sobre evaluación de la calidad. Bueno, no eran sobre evaluación de la calidad, eran sobre las expectativas de la calidad de los receptores o usuarios. Seguimos prácticamente sin modificar, yo creo que hemos añadido alguno y hemos suprimido algunos de los parámetros que sé que se incluían, pero sí, te puedo decir los que te he dicho anteriormente, que son los no verbales, también entraría el estilo, que está ahí entre medias, y luego estaría la emisión correcta como la gran estrella de los parámetros de calidad en la evaluación de la calidad y transmisión completa, aunque eso también últimamente ya se ha tendido a unificarlo, está la terminología... Y realmente creo que en total analizamos unos 10 u 11. Está también la gramática... Esos son los parámetros, digamos. Pero que el más importante sería la transmisión correcta del sentido y los otros lo acompañan y pueden modificar esa transmisión

Ángela Collados Ais

El estudiante tiene que aprender a evaluar y valorar adecuadamente su propia prestación...
CLINA

vol. 7-1, June 2021, 57-65

elSSN: 2444-1961

Ediciones Universidad de Salamanca - CC BY-NC-ND 
correcta, hasta tal punto que no llegue esa transmisión correcta, que no sea una transmisión correcta o maximizar esa transmisión correcta.

DDL: ¿Y crees que con esos 10 elementos, esos parámetros, se cubre todo lo que viene siendo el control de la calidad de la interpretación o crees que hay parámetros que no pueden evaluarse de ninguna manera?

ACA: Todos los parámetros son muy difíciles de evaluar. Seguramente que esos no son todos los parámetros de calidad, todo lo que se puede controlar en una interpretación. Porque claro que hay más elementos, como puede ser incluso el comportamiento con el micro que tenga el intérprete en una cabina, por ejemplo, los ruidos que se produzcan dentro de una cabina o la interacción incluso con el compañero. Es decir, hay muchísimos elementos que se deben tener en cuenta y luego, factores como pueden ser la técnica en general, etc. Es decir, estamos seguras de que estos 10 parámetros no cubren en su totalidad. Yo creo que la interpretación es un acto muy complejo y, por lo tanto, son muchas las variables que hay que tener en cuenta. Lo que pasa es que es bueno por lo menos aislar determinados parámetros para ir viendo la influencia de esos parámetros. Yo creo que se pueden introducir seguramente bastantes más parámetros. Luego, claro, hay que ver la forma en que se investigan los unos y otros. Eso dependerá del tipo de parámetro que se trate y luego claro, es que las situaciones son siempre muy diversas, es muy difícil, en realidad, si no se hace a nivel experimental evaluar precisamente esos parámetros de calidad en situación, se hace precisamente tan difícil porque son muy difíciles de aislar. Ya no sabemos achacar a qué parámetro, qué pérdida de valoración, por ejemplo, positiva.

DDL: ¿Y realmente crees que los parámetros se pueden aislar de todo, por ejemplo, de la cultura? Creo que la cultura es un factor muy importante que influye dentro del intérprete, y también dentro del resto de participantes en el acto de interpretación. Por eso me gustaría preguntarte si crees que hay alguna especie de asimetría cultural que sea especialmente notable y que influya tanto en la emisión como en la recepción, sobre todo del mensaje una vez interpretado.

ACA: Claro, el tema cultural está presente en todos los ámbitos. Nuestra investigación ha sido sobre todo en evaluación de la calidad de la interpretación de conferencias. Entonces, sí es cierto que en el caso de la interpretación de conferencias había casi menos diferencias culturales entre los asistentes, por ejemplo, a un determinado tipo especializado de congreso que en otros ámbitos, incluso aunque fuesen todos nacionales de un país. Es decir, igual hay más diferencias culturales entre españoles que provienen de un ámbito determinado, españoles que provienen de otro ámbito, etc. Entonces, eso es realmente difícil. Yo creo que de todas formas la interpretación simultánea es donde menos diferencias culturales puede haber en ese sentido. Donde sí se manifiestan más son en otras modalidades de interpretación, lógicamente. Quizá la

Ángela Collados Ais

El estudiante tiene que aprender a evaluar y valorar adecuadamente su propia prestación...
CLINA

vol. 7-1, June 2021, 57-65

elSSN: 2444-1961

Ediciones Universidad de Salamanca - CC BY-NC-ND 
más extrema se sitúa precisamente en la interpretación de los servicios públicos, interpretaciones de los tribunales también puede haber mucho más, en la interpretación de enlace pues depende de los ámbitos, pero quizá en el ámbito, como estoy diciendo, de la interpretación simultánea sea precisamente donde menos se produzcan esas diferencias. Por otra parte, claro, al ser la interpretación simultánea una interpretación que se produce con esa inmediatez simultánea, efectivamente, es muy difícil también para el intérprete tener en cuenta esas diferencias culturales. Si estamos hablando de diferencias culturales importantes, lógicamente, si son diferencias culturales que puede manejar en el propio discurso, pues se pueden solucionar, pero realmente si no, es bastante complicado. Es decir, técnicamente en cuanto a su realización es bastante complicado, pero también es muy complicado, pero tampoco existe tanta necesidad, diría yo, en el caso de la interpretación simultánea.

DDL: Y ahora que ha mencionado el sector público, ¿diría que existe un control de calidad sólido de interpretación en los servicios públicos, en juzgados, en hospitales o en centros educativos?

ACA: No, no lo creo. Para nada, no lo creo en absoluto. Y yo creo que esto es una cuestión que va lamentablemente y de forma ineludible unida al tema económico. Es decir, digamos que la interpretación simultánea es la estrella de todas las interpretaciones y por tanto ahí sí se puede permitir un cierto control de calidad y se hace, se realiza, de hecho. Porque quienes pagan, los grandes organizadores de congresos, del ámbito diplomático, etc., ahí se mueve dinero. Donde menos dinero se mueve, de manera evidente, es precisamente en este tipo de interpretaciones que se producen ante los servicios públicos, incluso ante los tribunales. Y realmente yo no estoy muy informada, este no es un tema que yo domine, pero claro lógicamente escuchamos todos cuál es la situación en la historia de la interpretación en los servicios públicos o la interpretación ante los tribunales. Es decir, que funcionan a base de contratas y subcontratas, etc. Claro que tiene que ver y es un tema que está claramente relacionado con la cuestión económica.

DDL: ¿Y cuál crees que debería ser la solución ante esta situación? Porque dado que la transposición de las directivas europeas como la directiva 2010-64 que versa solo de traducción e interpretación y que además también las leyes nacionales no cubren todo lo que viene siendo el concepto y la figura del intérprete en servicios públicos, ¿cuál crees que debería ser la solución?

ACA: Ahora mismo, la verdad, es que este es un tema bastante importante dentro de CCDUTI y sigue siendo un tema muy importante. De hecho, yo creo que hay grupos dentro de la CCDUTI que han seguido este tema con muchísimo ahínco, que han realizado una labor magnífica, precisamente para intentar modificarlo. Yo creo que la vía tiene que ser esa, a través de las Conferencias de Centros y Departamentos de

Ángela Collados Ais

El estudiante tiene que aprender a evaluar y valorar adecuadamente su propia prestación...
CLINA

vol. 7-1, June 2021, 57-65

elSSN: 2444-1961

Ediciones Universidad de Salamanca - CC BY-NC-ND 
Traducción e Interpretación del Estado español. A partir de ahí, los contactos que han tenido con la política, con el gobierno, con los propios tribunales, etcétera. Entonces la única vía es esa. Ahora, la solución que se proponga, yo creo que pasaría quizá por esa implicación y ese control a través de los centros y departamentos. Porque realmente un control externo no se va a producir, porque es lo que he dicho antes, no es un ámbito donde haya dinero, entonces no hay control, no va a haber un control externo. No va a haber el control externo que hay, por ejemplo, en la interpretación simultánea donde, simplemente, si no lo haces de una forma adecuada pues no te llaman otra vez. Al contrario, ahí se rige por el precio. Yo creo que la solución estaría o está en las acciones que se puedan emprender mediante todos los centros y departamentos de España. Y que sea esta asociación la que vea cuál sería la mejor modalidad para controlar esa calidad de la interpretación en ese ámbito. Se ha hablado de varias fórmulas, una ha sido ofrecer una serie de cursos a través de las propias universidades que certifiquen, que acrediten, otra sería a través de un examen, pero siempre con la participación de los centros y departamentos. La verdad es que no sé cuál sería la mejor vía. Pero, claro, tiene que producirse.

DDL: Cambiando de tema, en relación también con todo lo que es el ejercicio profesional de la interpretación, nos gustaría que nos pudiera comentar brevemente qué fallas se le ve al mercado de la interpretación actualmente en España, en cuanto a salarios o la subcontratación, precariedad laboral...

ACA: Bien, en cuanto al mercado general de la interpretación, últimamente no me muevo en el mercado de la interpretación, pero sí lo he conocido y lo he seguido. Yo creo que hay que diferenciar mucho las distintas modalidades. Es decir, la interpretación de conferencias sigue siendo una modalidad bien considerada y, por lo tanto, dentro del mercado, aunque estamos tendiendo cada vez más al inglés como como lingua franca, pues realmente creo que ahí las condiciones, sí se puede decir, son unas condiciones más adecuadas. No son en absoluto adecuadas en todo el resto del mercado, donde hay una zona gris incluso. Con las contratas y las subcontratas, lo que hemos hablado, no sabría qué más añadir. Es evidente que hay una diferencia muy clara entre la interpretación de conferencias y el resto de las modalidades de interpretación.

DDL: Se puede decir, quizá, que en la interpretación de conferencias hay más demanda y por eso también quizás mejores condiciones o más regulación.

ACA: Yo no creo que sea la mayor demanda lo que lo haga, sino la calidad de la demanda. La calidad de la demanda, que es lo que he mencionado un poco antes con otras palabras, entre comillas quiero decir, porque la calidad de la demanda es una demanda económicamente potente, a eso me refiero con la calidad de la demanda. Mientras que en el otro ámbito, esa «calidad de la demanda» no es así. Quizá, porque

Ángela Collados Ais

El estudiante tiene que aprender a evaluar y valorar adecuadamente su propia prestación...
CLINA

vol. 7-1, June 2021, 57-65

elSSN: 2444-1961

Ediciones Universidad de Salamanca - CC BY-NC-ND 
los usuarios de ese tipo de interpretación, evidentemente, no tienen un poder económico fuerte y porque las instituciones no se preocupan lo suficiente, porque precisamente lo necesitan. Es el tipo de interpretación, quizá, más útil para la sociedad, por lo menos para las partes más desfavorecidas de la sociedad.

DDL: Y bueno, siguiendo la línea del control de calidad y pasando un poco a la didáctica de la interpretación, que es otra de tus especialidades, ¿crees que se debe enseñar a realizar un buen control de calidad en interpretación a los estudiantes de formación universitaria?

ACA: Sí, que ellos aprendan a evaluar, el control de calidad dentro de la enseñanza, de la carrera. Yo creo que sí, que ese control de calidad lo tenemos que asumir nosotros mismos, los propios intérpretes, y entonces yo creo que eso forma parte clarísimamente de lo que es una enseñanza de interpretación. El estudiante tiene que aprender a evaluar y valorar adecuadamente su propia prestación, al margen ya de las calificaciones que se le otorguen en las distintas asignaturas, que deben ser asumidas también por los propios estudiantes, porque si no no tiene sentido. Que el estudiante sepa por qué esto ha supuesto una bajada de puntos o no ha supuesto una bajada de la nota. Pero para eso es muy importante que ellos mismos ya sepan aplicar esa hoja de evaluación, que son las que se aplican luego en las propias asignaturas. Entonces yo sí creo que es muy importante y creo que es importante que lo hagan de forma casi metódica, que por lo menos, no en todas las clases evidentemente, pero sí periódicamente, ellos evalúen su propia prestación. Por ejemplo, ahora mismo en el máster de Interpretación de Conferencias, que el único público que yo sepa que hay en el territorio español, pues en este máster una de las principales tareas del estudiante no es tanto que haga una interpretación perfecta, sino que haga una buena evaluación de su propia interpretación, de su propia prestación. Porque realmente esa es la única forma de mejorar y de que finalmente sean interpretaciones de calidad, es decir, lo importante es el proceso que determina el producto.

DDL: Para ir terminando y respondiéndote un poco a lo que querías ejemplificar, en nuestra facultad, aquí en la Facultad de Traducción y Documentación en Salamanca, realizamos una prueba de autodiagnóstico, tanto en interpretación consecutiva como en la introducción a la interpretación simultánea, en la que, como dices, cada alumno debe evaluar su propia prestación y lo importante es cómo se evalúa, digamos el método de evaluación. Supongo que esto es precisamente a lo que te referías, ¿no?

ACA: Sí, eso. Y sobre todo decir que evalúe el proceso, el resultado del producto pero a través del proceso. Por ejemplo, ¿por qué se ha producido ese error? O, ¿por qué se ha producido esa omisión que sí era importante? Y ¿cómo se podría solucionar, por qué se ha producido y cómo se podría haber evitado? Precisamente para ir introduciendo nuevas posibilidades de cara al futuro, porque si no nos quedaríamos

Ángela Collados Ais

El estudiante tiene que aprender a evaluar y valorar adecuadamente su propia prestación...
CLINA

vol. 7-1, June 2021, 57-65

elSSN: 2444-1961

Ediciones Universidad de Salamanca - CC BY-NC-ND 
simplemente en que una interpretación hoy está bien, mañana está mal, pero realmente lo importante es llegar al por qué se producen determinados fallos también positivamente, no solamente se analizan los fallos. Se maximizan aquellas estrategias que los estudiantes han aprendido y han aplicado perfectamente. Yo creo que en una evaluación no se trata solamente de evaluar aquello que no ha funcionado, sino también aquello que ha funcionado estupendamente, para reforzarlo.

DDL: ¿Recomiendas algún otro ejercicio para reflexionar sobre la propia interpretación a los alumnos, para que ellos puedan hacer un control de calidad de su trabajo que no sea el autodiagnóstico?

ACA: Yo creo que es muy interesante, por ejemplo, en las propias clases, el intercambio de interpretaciones. Que un alumno no siempre evalúe su propia prestación, sino que evalúe la prestación del compañero, tal y cómo evaluaría la suya propia.

DDL: Interesante.

ACA: Creo que eso es muy importante, porque además siempre corremos el riesgo de que seamos los mismos evaluadores, es decir, el propio estudiante en la autoevaluación y el profesor. El ir intercambiando es bueno para que haya otra evaluación diferente que no sea la que he mencionado, de dos actores, pero también es importante para que ese mismo estudiante perciba determinados elementos que si no no hubiese percibido, tanto en positivo como en negativo. Pero sobre todo ahí en positivo.

DDL: Nos ha parecido muy interesante tu aportación y la verdad es que se nos ha ido volando el tiempo y tenemos que terminar la entrevista. Muchas gracias, Ángela.

ACA: De verdad que ha sido un placer para mí y muchísimas gracias a vosotros. Mi primera idea era haber sido periodista, con lo cual pues mucha envidia de que estéis ahí. Creo que la labor que estás haciendo es estupenda. Enhorabuena.

\section{Entrevista: «Don de Lenguas»}

Transcripción: Beatriz Guerrero García

Enlace: https://www.ivoox.com/entrevista-a-angela-collados-ais-audios-mp3_rf_25730025_1.html

Duración: 26:51:00

Ángela Collados Ais

El estudiante tiene que aprender a evaluar y valorar adecuadamente su propia prestación... 


\title{
NOTA BIOGRÁFICA
}

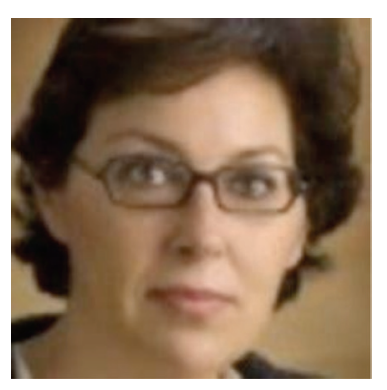

Diplomada en Traducción e Interpretación (especialidad Interpretación), licenciada en Derecho y doctora en Interpretación. Es intérprete de conferencias y docente de Interpretación en la Facultad de Traducción e Interpretación de la Universidad de Granada desde 1987, donde imparte interpretación de enlace y de conferencias en la combinación alemán-español. Ha dirigido diversos proyectos de investigación y es la investigadora responsable del grupo de investigación ECIS (Evaluación de la Calidad en Interpretación Simultánea), activo desde 1995. Sus publicaciones se centran fundamentalmente en el ámbito de la calidad y la docencia de la Interpretación.

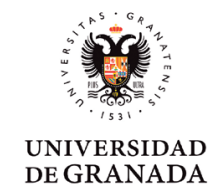

\author{
Ángela Collados: \\ https://orcid.org/0000-0001-8417-0717
}

\section{BIBLIOGRAFÍA}

Collados Aís, Á., Pradas Macías, E,M., Stévaux, E. y García Becerra, O. (2007). La evaluación de la calidad en interpretación simultánea: Parámetros de incidencia. Comares: Granada.

Collados Aís, Á. y Fernández Sánchez, M.M. (2001). Manual de Interpretación Bilateral. Comares: Granada.

Collados Aís, Á. (1998). La evaluación de la calidad en interpretación simultánea. La importancia de la comunicación no verbal. Comares: Granada.

Ángela Collados Ais

El estudiante tiene que aprender a evaluar y valorar adecuadamente su propia prestación... 